\title{
Srinivasa Ramanujan: Going Strong at 125, Part II
}

\section{Krishnaswami Alladi, Editor}

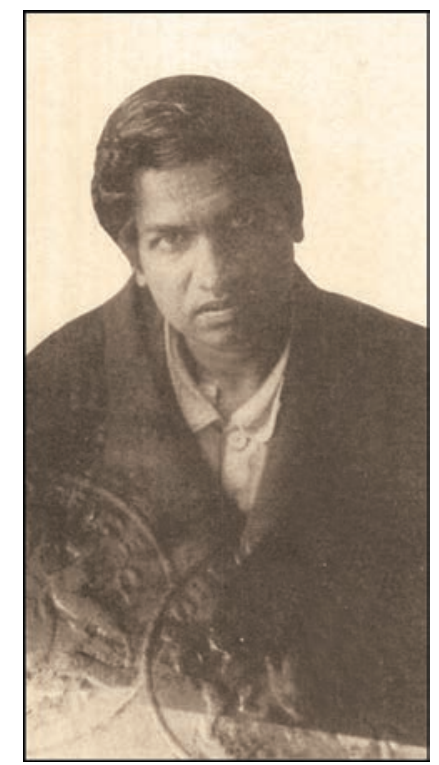

Ramanujan passport photo.

The 125th anniversary of the birth of Srinivasa Ramanujan was on December 22, 2012. To mark the occasion, the Notices is publishing a feature article of which this is the second and final installment. This installment contains pieces by Ken Ono, Kannan Soundararajan, Robert Vaughan, and Ole Warnaar on various aspects of Ramanujan's work. The first installment appeared in the December 2012 issue, and contained an introductory piece by Krishnaswami Alladi plus pieces by George Andrews, Bruce Berndt, and Jonathan Borwein on Ramanujan's work.

Krishnaswami Alladi is professor of mathematics at the University of Florida. His email address is a11adik@uf1. edu.

DOI: http://dx.doi.org/10.1090/noti926

\section{Ken Ono}

\section{Modular Forms, Partitions, and Mock Theta Functions}

Ramanujan's work on modular forms, partitions, and mock theta functions is rich with tantalizing examples of deeper mathematical structures. Indeed, his tau-function, his partition congruences, and his mock theta functions are prototypes that have helped to shape the modern mathematical landscape.

Ramanujan was fascinated by the coefficients of the function

$$
\text { (1) } \begin{aligned}
\Delta(z) & =\sum_{n=1}^{\infty} \tau(n) q^{n}:=q \prod_{n=1}^{\infty}\left(1-q^{n}\right)^{24} \\
& =q-24 q^{2}+252 q^{3}-1472 q^{4}+\cdots,
\end{aligned}
$$

where $q:=e^{2 \pi i z}$ and $\operatorname{Im}(z)>0$. This function is a weight 12 modular form. In other words, $\Delta(z)$ is a function on the upper half of the complex plane such that

$$
\Delta\left(\frac{a z+b}{c z+d}\right)=(c z+d)^{12} \Delta(z)
$$

for every matrix $\left(\begin{array}{ll}a & b \\ c & d\end{array}\right) \in \mathrm{SL}_{2}(\mathbb{Z})$. He conjectured (see p. 153 of [12]) that

$$
\tau(n m)=\tau(n) \tau(m),
$$

for every pair of coprime positive integers $n$ and $m$, and that

$$
\tau(p) \tau\left(p^{s}\right)=\tau\left(p^{s+1}\right)+p^{11} \tau\left(p^{s-1}\right),
$$

for primes $p$ and positive integers $s$. Mordell proved these conjectures, and in the 1930s Hecke later

Ken Ono is the Asa Griggs Candler Professor of Mathematics at Emory University. His email address is ono@mathcs. emory. edu. He thanks the NSF for their generous support. 
developed a framework for the theory of modular forms and $L$-functions in which such properties play a fundamental role.

Ramanujan was also very interested in the size of the numbers $\tau(n)$, and for primes $p$ he conjectured (see pp. 153-154 of [12]), but could not prove, that

$$
|\tau(p)| \leq 2 p^{\frac{11}{2}} .
$$

This speculation is the first example of the Ramanujan-Petersson Conjectures, among the deepest problems in the analytic theory of automorphic forms. This inequality was triumphantly confirmed by Deligne [8] as a corollary to his proof of the Weil Conjectures, work which won him a Fields Medal in 1978. Although Ramanujan did not anticipate the Weil Conjectures (the Riemann Hypothesis for varieties over finite fields), he correctly anticipated the importance of optimally bounding coefficients of modular forms.

Ramanujan is also well known for his work on the congruence properties of his tau-numbers. For example, he proved that (see page 159 of [12])

$$
\tau(n) \equiv \sum_{d \mid n} d^{11} \quad(\bmod 691)
$$

About forty years ago, Serre [13] and SwinnertonDyer [14] wrote beautiful papers interpreting such congruences in terms of certain two-dimensional $\ell$-adic representations of $\mathrm{Gal}(\overline{\mathbb{Q}} / \mathbb{Q})$, the Galois group of the algebraic closure of $\mathbb{Q}$. Deligne had just proved that such representations encode the coefficients of certain modular forms as "traces of the images of Frobenius elements". Serre and Swinnerton-Dyer interpreted Ramanujan's taucongruences, such as (2), as the first nontrivial examples of certain exceptional representations. For the prime $\ell=691$, there is a (residual) Galois representation

$$
\rho_{\Delta, 691}: \operatorname{Gal}(\overline{\mathbb{Q}} / \mathbb{Q}) \longrightarrow \mathrm{GL}_{2}(\mathbb{Z} / 691 \mathbb{Z})
$$

which, for primes $p \neq 691$, satisfies

$$
\rho_{\Delta, 691}(\operatorname{Frob}(p))=\left(\begin{array}{cc}
1 & * \\
0 & p^{11}
\end{array}\right),
$$

where $\operatorname{Frob}(p) \in \operatorname{Gal}(\overline{\mathbb{Q}} / \mathbb{Q})$ denotes the "Frobenius element at $p$ ". Congruence (2) is then implied by Deligne's prescription that, for primes $p \neq 691$, one has

$\tau(p)=\operatorname{Tr}\left(\rho_{\Delta, 691}(\operatorname{Frob}(p))\right) \equiv 1+p^{11} \quad(\bmod 691)$.

The theory of modular $\ell$-adic Galois representations has subsequently flourished and famously is the "language" of Wiles's proof of Fermat's Last Theorem.

Ramanujan's work on tau-congruences is intimately related to his work on partition congruences. A partition of the natural number $n$ is any nonincreasing sequence of natural numbers whose sum is $n$, and the number of partitions of $n$ is denoted by $p(n)$. The generating function for $p(n)$ has two convenient representations:

(3)

$$
\begin{aligned}
\sum_{n=0}^{\infty} p(n) q^{n} & =\prod_{n=1}^{\infty} \frac{1}{1-q^{n}} \\
& =1+\sum_{n=1}^{\infty} \frac{q^{n^{2}}}{(1-q)^{2}\left(1-q^{2}\right)^{2} \cdots\left(1-q^{n}\right)^{2}} .
\end{aligned}
$$

The infinite product representation is analogous to the infinite product for $\Delta(z)$ in (1), which explains the important role that modular forms play in the study of $p(n)$, while the other representation as a "basic hypergeometric series" foreshadows Ramanujan's last work: his discovery of "mock theta functions".

In groundbreaking work, Ramanujan proved that

$$
\begin{aligned}
p(5 n+4) \equiv 0 & (\bmod 5), \\
p(7 n+5) \equiv 0 & (\bmod 7), \\
p(11 n+6) \equiv 0 & (\bmod 11) .
\end{aligned}
$$

He also conjectured corresponding congruences, known as Ramanujan's congruences, modulo powers of 5, 7, and 11. Extending his ideas, Atkin and Watson proved [3], [15]:

$$
\begin{aligned}
\text { If } \delta=5^{a} 7^{b} 11^{c} \text { and } 24 \lambda & \equiv 1 & (\bmod \delta), \\
\text { then } p(\delta n+\lambda) & \equiv 0 & \left(\bmod 5^{a} 7^{\left\lfloor\frac{b}{2}\right\rfloor+1} 11^{c}\right) .
\end{aligned}
$$

These congruences have inspired much further work.

In the 1940s Dyson, seeking a combinatorial explanation for the congruences, defined [9] the rank of a partition to be the largest summand minus the number of summands. He conjectured that the partitions of $5 n+4$ (resp. $7 n+5)$ are divided into 5 (resp. 7) groups of equal size when sorted by their ranks modulo 5 (resp. 7), thereby providing a combinatorial explanation for the congruences mod 5 and 7 . This conjecture was proved in the 1950s by Atkin and Swinnerton-Dyer [5]. Dyson was unable to offer a combinatorial explanation for the mod 11 congruence, and he conjectured the existence of a statistic, which he referred to as the "crank", which would suitably explain the congruences mod 5, 7, and 11. In the 1980s Andrews and Garvan [2] finally found the elusive crank.

In addition to finding a combinatorial explanation for Ramanujan's original congruences, researchers have looked for further congruences. In the 1960s Atkin [4] found congruences, though not so systematic, with modulus $13,17,19,23$, 29 , and 31. About ten years ago the author and Ahlgren [1], [10] found that there are partition 


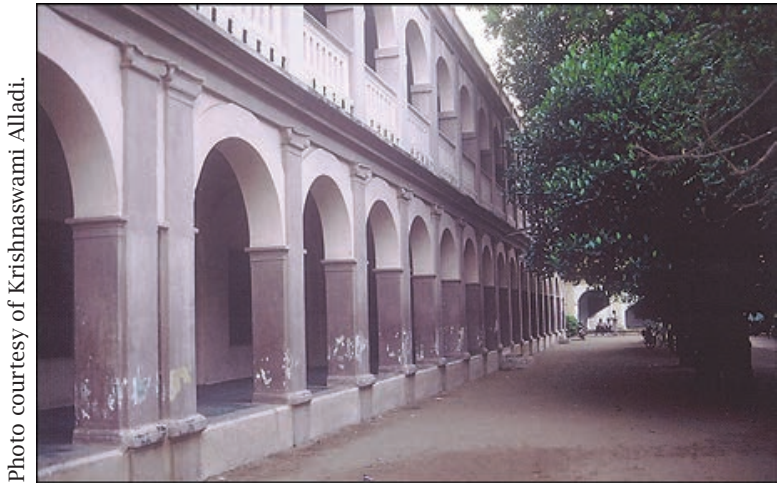

The lovely colonial style architecture is evident in the Town High School in Kumbakonam that Ramanujan attended.

congruences modulo every integer $Q$ relatively prime to 6. For example, we have

$$
p\left(59^{4} \cdot 13 n+111247\right) \equiv 0 \quad(\bmod 13) .
$$

These more recent results arise from the fact that (3) is essentially a modular form. This fact, combined with deep work of Shimura, makes it possible to apply the methods of Deligne and Serre, which explained Ramanujan's tau-congruences, to the partition numbers.

During his last year of life, when he was seeking a return to good health in south India, Ramanujan discovered functions he called mock theta functions. In his last letter to Hardy, dated January 12, 1920, Ramanujan shared hints (see p. 220 of [6]) of his last theory. The letter, roughly four typewritten pages, consists of formulas for seventeen strange power series and a discussion of their asymptotics. It contained no proofs of any kind. By changing signs in (3), we obtain a typical example of a mock theta function:

$$
\begin{aligned}
f(q) & =\sum_{n=0}^{\infty} a_{f}(n) q^{n} \\
& :=\sum_{n=0}^{\infty} \frac{q^{n^{2}}}{(1+q)^{2}\left(1+q^{2}\right)^{2} \cdots\left(1+q^{n}\right)^{2}} .
\end{aligned}
$$

For followers of Ramanujan, the main questions were: What did Ramanujan mean by a mock theta function, and what roles do these functions play in mathematics? These questions would remain open for eighty years. In the meantime, such researchers as Andrews, Choi, Dragonette, Dyson, Gordon, Hickerson, McIntosh, Selberg, and Watson, to name a few, proved beautiful theorems about these strange $q$-series. Then in 2002, in his Ph.D. thesis [17] written under Zagier, Zwegers made sense of the mock theta functions. He discovered how to "complete" them by adding a nonholomorphic function, a so-called "period integral", to obtain a real analytic modular form.
In the case of $f(q)$ and its companion $\omega(q)$, Zwegers defined the vector-valued functions

$$
\begin{aligned}
F(z) & =\left(F_{0}(z), F_{1}(z), F_{2}(z)\right)^{T} \\
& :=\left(q^{-\frac{1}{24}} f(q), 2 q^{\frac{1}{3}} \omega\left(q^{\frac{1}{2}}\right), 2 q^{\frac{1}{3}} \omega\left(-q^{\frac{1}{2}}\right)\right)^{T}, \\
G(z) & =\left(G_{0}(z), G_{1}(z), G_{2}(z)\right)^{T} \\
& :=2 i \sqrt{3} \int_{-\bar{z}}^{i \infty} \frac{\left(g_{1}(\tau), g_{0}(\tau),-g_{2}(\tau)\right)^{T}}{\sqrt{-i(\tau+z)}} d \tau,
\end{aligned}
$$

where the $g_{i}(z)$ are theta functions, and he proved that $H(z):=F(z)-G(z)$ satisfies

$$
H(z+1)=\left(\begin{array}{ccc}
\zeta_{24}^{-1} & 0 & 0 \\
0 & 0 & \zeta_{3} \\
0 & \zeta_{3} & 0
\end{array}\right) H(z)
$$

and

$$
H(-1 / z)=\sqrt{-i z} \cdot\left(\begin{array}{ccc}
0 & 1 & 0 \\
1 & 0 & 0 \\
0 & 0 & -1
\end{array}\right) H(z),
$$

where $\zeta_{n}:=e^{\frac{2 \pi i}{n}}$, making it a vector-valued real analytic modular form.

All of Ramanujan's mock theta functions turn out to be holomorphic parts of special weight $1 / 2$ real analytic modular forms, which Bruinier and Funke [7] call weak harmonic Maass forms. Loosely speaking, a weight $k$ harmonic Maass form is a smooth function $M(z)$ on $\mathbb{\square}$ which transforms as does a weight $k$ modular form and which also satisfies $\Delta_{k}(M)=0$. Here the hyperbolic Laplacian $\Delta_{k}$, where $z=x+i y \in \mathbb{U}$ with $x, y \in \mathbb{R}$, is given by

$$
\Delta_{k}:=-y^{2}\left(\frac{\partial^{2}}{\partial x^{2}}+\frac{\partial^{2}}{\partial y^{2}}\right)+i k y\left(\frac{\partial}{\partial x}+i \frac{\partial}{\partial y}\right) .
$$

Since modular forms appear prominently in mathematics, I think that one expects these functions to have far-reaching implications. One might expect the functions themselves to play many roles. This has already turned out to be the case. These forms have appeared prominently in the following subjects [11], [16]: partitions and $q$-series, moonshine, Donaldson invariants, probability theory, Borcherds products and elliptic curves, and many others.

\section{References}

[1] S. AHLGRen and K. ONO, Congruence properties for the partition function, Proc. Nat. Acad. Sci. U.S.A. 98 (2001), 12882-12884.

[2] G. E. ANDREWS and F. GARVAN, Dyson's crank of a partition, Bull. Amer. Math. Soc. (N.S.) 18, no. 2 (1988), 167-171.

[3] A. O. L. AtKIN, Proof of a conjecture of Ramanujan, Glasgow J. Math. 8 (1967), 14-32.

[4] __ Multiplicative congruence properties and density problems for $p(n)$, Proc. London Math. Soc. 18 (1968), 563-576.

[5] A. O. L. AtKin and H. P. F. Swinnerton-Dyer, Some properties of partitions, Proc. London Math. Soc. 66 , no. 4 (1954), 84-106. 
[6] B. C. BERNDT and R. A. RANKIN, Ramanujan: Letters and Commentary, History of Mathematics, vol. 9, Amer. Math. Soc., Providence, RI, 1995.

[7] J. H. BRUINIER and J. FunKE, On two geometric theta lifts, Duke Math. J. 125 (2004), 45-90.

[8] P. Deligne, La conjecture de Weil. I, Inst. Hautes Études Sci. Publ. Math., no. 43 (1974), 273-307.

[9] F. Dyson, Some guesses in the theory of partitions, Eureka (Cambridge) 8 (1944), 10-15.

[10] K. ONO, Distribution of the partition function modulo m, Ann. Math. 151 (2000), 392-307.

[11] _ Unearthing the Visions of a Master: Harmonic Maass Forms and Number Theory, 2008 HarvardMIT Current Developments in Mathematics, Int. Press, Somerville, MA, 2009, pp. 347-454.

[12] S. Ramanujan, Collected Papers of Ramanujan, Cambridge Univ. Press, Cambridge, 1927.

[13] J.-P. SERRE, Congruences et forms modulaires (d'pres̀ $H$. P. F. Swinnerton-Dyer), Sèminaire Bourbaki, 24e année (1971/1972), Exp. No. 416, Lecture Notes in Math., vol. 317, Springer, 1973, pp. 319-338.

[14] , On $\ell$-adic representations and congruences for coefficients of modular forms, Modular Functions of One Variable, III (Proc. Internat. Summer School, Univ. Antwerp, 1972), Lecture Notes in Math., vol. 350, Springer, 1973, pp. 1-55.

[15] G. N. WATson, Ramanujan's Vermutung über Zerfällungsanzahlen, J. Reine Angew. Math. 179 (1938), 97-128.

[16] D. ZAGIER, Ramanujan's Mock Theta Functions and Their Applications [d'après Zwegers and BringmannOno], Sem. Bourbaki, 60ème année, 2006-2007, no. 986.

[17] S. P. ZwEGERS, Mock theta functions, Ph.D. thesis, Universiteit Utrecht, 2002.

\section{K. Soundararajan}

\section{Ramanujan and the Anatomy of Integers}

The story of Hardy and Ramanujan and the taxicab number 1729 is well known. It may be less familiar that taxicab numbers also formed the motivation for a famous paper of Hardy and Ramanujan on the number of prime factors of integers (see paper 35 and announcement 32 in [22]). They note that "anybody who will make a practice of factorising numbers, such as the numbers of taxi-cabs" would verify that "round numbers"-integers composed of a considerable number of comparatively small factors-“are exceedingly rare." Hardy and Ramanujan explain this phenomenon by showing that almost all numbers $n$ below $x$ (in the sense of density) are formed of about $\log \log n$ prime factors. More precisely, if $\omega(n)$ denotes the number of distinct prime factors of $n$, then $|\omega(n)-\log \log n| \leq \Phi(n) \sqrt{\log \log n}$ for almost all $n \leq x$, where $\Phi(n)$ is any function tending to infinity with $n$, and the same result holds also for

K. Soundararajan is professor of mathematics at Stanford University. His email address is ksound@stanford.edu.

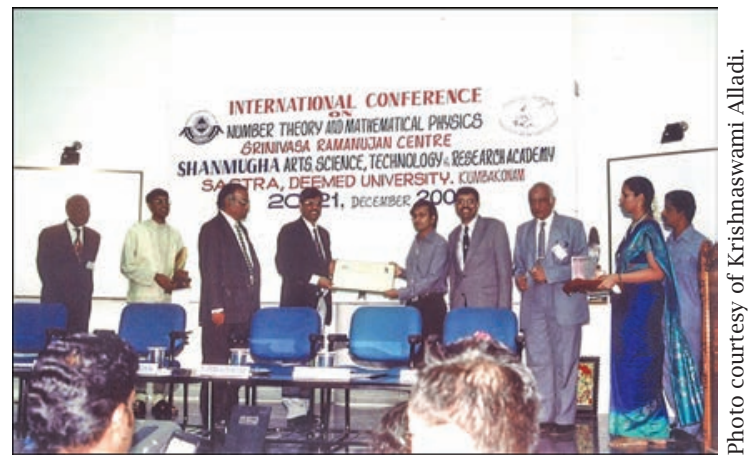

Kannan Soundarajan receiving the First SASTRA Ramanujan Prize from Dr. Aurobinda Mitra, director of the Department of Science and Technology, India, on December 20, 2005. Manjul Bhargava (second from left), also a winner of the prize that year, looks on. Also in the picture are Krishnaswami Alladi, chair of the prize committee (next to Soundararajan), and R. Sethuraman, vice-chancellor of SASTRA (between Bhargava and Mitra).

$\Omega(n)$, which counts the prime factors of $n$ with multiplicity.

The Hardy-Ramanujan result was refined by Erdős and Kac [9], who showed that $(\omega(n)-$ $\log \log n) / \sqrt{\log \log n}$ has an approximately normal distribution with mean 0 and variance 1 . The ErdósKac theorem is in relation to the Hardy-Ramanujan theorem as the Central Limit Theorem is to the Law of Large Numbers. These two results formed the impetus for the development of probabilistic ideas in number theory, and we refer the reader to Elliott [7], Tenenbaum [27], and Kubilius [17] for accounts of related work.

Hardy and Ramanujan proved their theorem by means of an ingenious induction argument that establishes a uniform upper bound for $\pi_{k}(x)$, the number of integers below $x$ with exactly $k$ prime factors. The prime number theorem tells us that $\pi(x)=\pi_{1}(x)$, the number of primes below $x$, is approximately $x / \log x$. Landau generalized this to show that, for any fixed $k$ and large $x, \pi_{k}(x)$ is approximately $\frac{x}{\log x} \frac{(\log \log x)^{k-1}}{(k-1) !}$. In probabilistic terms, this suggests that the number of prime factors of an integer has an approximately Poisson distribution with parameter $\log \log x$. Since a Poisson distribution with large parameter $\lambda$ approximates a normal distribution with mean and variance equal to $\lambda$, this interpretation is in keeping with the Hardy-Ramanujan and Erdős-Kac theorems. It is therefore natural to wonder whether the uniform upper bound of Hardy and Ramanujan could be refined to an asymptotic formula, which would be a considerable strengthening of the Erdős-Kac theorem. This problem was highlighted by both 
Hardy and Ramanujan and inspired a great deal of work over the years by many authors (notably Pillai [20], Erdős [8], Sathe [25], and Selberg [26]), culminating in the definitive work of Hildebrand and Tenenbaum [15].

The Hardy-Ramanujan theorem, furthered by the insight of Erdős, led to a host of problems (see, for example, [13]) designed to understand the multiplicative structure of a random integer-or as Granville [4] has termed it, "the anatomy of (random) integers". Interestingly, the anatomy of a random integer is strikingly similar to the anatomy of random polynomials over finite fields and to the anatomy of the cycle decompositions of random permutations; see [2] and the forthcoming graphic novel(!) [12] for accounts of these similarities. Moreover, understanding the anatomy of integers has proven valuable in many problems of algorithmic number theory, for example, in the development of factorization algorithms such as the quadratic sieve [21].

Out of the many beautiful theorems in the area, let us mention the striking recent work of Ford [10] in understanding the distribution of integers having a divisor of a given size. Ford's work resolved a longstanding problem of Erdős: How many distinct integers are there in the $N \times N$ multiplication table? The Hardy-Ramanujan theorem, Erdôs observed, gives a quick proof that the number of distinct integers in the multiplication table is $o\left(N^{2}\right)$. A typical integer of size $N$ has about $\log \log N$ prime factors, and so the product of two such integers has about $2 \log \log N$ prime factors. Thus a typical element appearing in the multiplication table has $2 \log \log N$ prime factors, whereas a typical integer of size $N^{2}$ has only about $\log \log \left(N^{2}\right)=\log \log N+\log 2$ prime factors. In other words, elements in the multiplication table are quite atypical and thus few in number. Refining a result of Tenenbaum [28], Ford's work establishes that true order of magnitude for the number of integers in the multiplication table is $N^{2} /\left((\log N)^{\delta}(\log \log N)^{\frac{3}{2}}\right)$, with $\delta$ being the esoteric constant $1-(1+\log \log 2) / \log 2=$ $0.086 \ldots$ In the study of random permutations, there arises an analogous problem: Given $n$ and $1 \leq k \leq n-1$, the permutation group $S_{n}$ acts naturally on the $k$-element subsets of $\{1, \ldots, n\}$. Now given a random element in $S_{n}$, what is the probability that there exists some $k$-element subset left fixed by this permutation? Partial results on this problem were established by Luczak and Pyber [19] and have interesting applications [5]. Using Ford's work on integers as a guide to the anatomy of permutations, one can obtain a more refined understanding of this question [6].

We now turn to Ramanujan's work on "highly composite numbers". These are numbers $n$ such that $d(n)>d(k)$ for all $1 \leq k<n$, where $d(n)$ counts the number of divisors of $n$. Ramanujan described (see paper 15 in [22]) the anatomy of such highly composite integers and also made a careful study of the extremal order of $d(n)$. Ramanujan was also interested in finding large values of the iterated functions $d(d(n)$ ) (whose exact order is determined in the surprisingly recent paper [3]) and $d(d(d(n)))$. Closely related to highly composite numbers are the superabundant numbers: these are the integers $n$ for which $\sigma(n) / n$ is larger than $\sigma(k) / k$ for all $1 \leq k \leq n-1$, and where $\sigma(n)$ denotes the sum of the divisors of $n$. Superabundant numbers were studied extensively by Alaoglu and Erdős [1], who were directly motivated by Ramanujan's work. Interestingly, Ramanujan himself had studied these classes of numbers, in notes related to his long paper on highly composite numbers, but owing to space considerations these were not included in the published work. These manuscript notes were discovered as part of Ramanujan's "Lost Notebook" [23] and were published with annotations by Nicolas and Robin in [24]. While interesting, these problems seem far from the mainstream, and so it may come as a surprise that the Riemann Hypothesis is equivalent to the estimate $\sigma(n) \leq H_{n}+\exp \left(H_{n}\right) \log H_{n}$ (where $H_{n}$ is the "harmonic number" $\left.H_{n}=\sum_{j=1}^{n} 1 / j\right)$, and the difficult cases of this inequality are precisely when $n$ is a superabundant number; see Lagarias [18] for a charming account of this connection.

We end by mentioning an unpublished and largely unknown fragment of Ramanujan on a central topic in the anatomy of integers, namely, on "smooth" (or "friable") numbers. A number is called $y$-smooth if all its prime factors are at most $y$. Smooth numbers appear prominently in algorithmic number theory (e.g., in factoring algorithms) and are also useful in seemingly unrelated questions such as Waring's problem; see [16] and [11] for excellent surveys on these integers without large prime factors. A basic question is to estimate $\Psi(x, y)$, which counts the number of $y$ smooth integers below $x$; a precise understanding of $\Psi(x, y)$ uniformly in a wide range is equivalent to the Riemann Hypothesis [14]. The first published work on smooth numbers in 1930 was by Dickman, who showed that if $x=y^{u}, u>0$ is considered fixed, and $y$ tends to infinity, then $\Psi(x, y) \sim \rho(u) x$, where $\rho$ (the Dickman function) is defined as the unique continuous solution to the differentialdifference equation $u \rho^{\prime}(u)=-\rho(u-1)$ for $u>1$, and with initial condition $\rho(u)=1$ for $0 \leq u \leq 1$. Given the significance of smooth numbers, it may be interesting to record that Ramanujan appears to have made a study of this problem. On page 337 of [23] one finds formulas for $\Psi\left(y^{u}, y\right)$ in the range $1 \leq u \leq 5$, which give inclusion-exclusion type 
expressions for $\rho(u)$ in terms of iterated integrals and with a clear indication of how the pattern is to be continued.

\section{References}

[1] L. AlAOGLU and P. ERDŐS, On highly composite and similar numbers, Trans. Amer. Math. Soc. 56 (1944) 448-469.

[2] R. Arratia, A. D. BARbour, and S. TAVARÉ, Random combinatorial structures and prime factorizations, Notices Amer. Math. Soc. 44 (1997), 903-910.

[3] Y. Buttkewitz, C. Elsholtz, K. Ford, and J-C. SCHLAGE-PUCHTA, A problem of Ramanujan, Erdős and Kátai on the iterated divisor function, Int. Math. Res. Notices, to appear.

[4] J. M. De KonincK, A. GRANVILle, and F. LuCA (eds.), Anatomy of Integers, CRM Proceedings and Lecture Notes, Vol. 46, Amer. Math. Soc., Providence, RI, 2008.

[5] P. Diaconis, J. Fulman, and R. Guralnick, On fixed points of permutations, J. Algebr. Comb. 28 (2008) 189-218.

[6] P. Diaconis and K. Soundararajan, Unpublished notes on fixed sets of random permutations (2012).

[7] P. D. T. A. ElLIOTT, Probabilistic Number Theory (2 volumes), Grund. Math. Wiss., vol. 239 and 240, Springer Verlag, 1979 and 1980.

[8] P. ERDŐs, Integers having exactly $k$ prime factors, Ann. Math. 49 (1948), 53-66.

[9] P. ERDŐs and M. KAC, The Gaussian law of errors in the theory of additive number theoretic functions, Amer. J. Math. 62 (1940), 738-742.

[10] K. FORD, The distribution of integers with a divisor in a given interval, Annals of Math. 168 (2008), 367-433.

[11] A. GRANVILLE, Smooth numbers: computational number theory and beyond, in Algorithmic Number Theory: Lattices, Number Fields, Curves and Cryptography, MSRI Publications, Vol. 44, Cambridge Univ. Press, 2008 pp. 267-323.

[12] A. GRANVILLE and J. GRANVILLE, MSI: Anatomy of integers and permutations (graphic novel, art by Robert J. Lewis, cover by Spain Rodriguez), Princeton University Press, forthcoming (2013).

[13] R. R. Hall and G. Tenenbaum, Divisors, Cambridge Tracts in Math., vol. 90, Cambridge Univ. Press, 1988.

[14] A. HILDEBRAND, Integers free of large prime factors and the Riemann hypothesis, Mathematika 31 (1984), $258-271$

[15] A. Hildebrand and G. Tenenbaum, On the number of prime factors of an integer, Duke Math. J. 56 (1988), 471-501.

[16] _ Integers without large prime factors, J. Théorie des Nombres Bordeaux 5 (1993), 411-484.

[17] J. KubILIUS, Probabilistic methods in the theory of numbers, Trans. Math. Monographs, vol. 11, Amer. Math. Soc., 1964.

[18] J. LAGARIAS, An elementary problem equivalent to the Riemann Hypothesis, Amer. Math. Monthly 109 (2002), 534-543.

[19] T. LUCZAK and L. PYBER, On random generation of the symmetric group, Comb. Probab. Comput. 2 (1993), 505-512.

[20] S. S. PILLAI, On the number of numbers which contain a fixed number of prime factors, Math. Student (1929), $250-251$.
[21] C. Pomerance, A tale of two sieves, Notices Amer. Math. Soc. 43 (1996), 1473-1485.

[22] S. Ramanujan, Collected Papers of Srinivasa Ramanujan (G. H. Hardy, P. V. Seshu Aiyar, and B. M. Wilson, eds.), AMS Chelsea Publishing, Third Printing 1999.

[23] _ The Lost Notebook and Other Unpublished Papers, Narosa, New Delhi, and Springer-Verlag, Berlin, 1988.

[24] _ Highly composite numbers, annotated and with a foreword by J-L Nicolas and G. Robin, Ramanujan J. 1 (1997) 119-153.

[25] L. G. SATHE, On a problem of Hardy on the distribution of integers having a given number of prime factors. I-IV, J. Indian Math. Soc. 17 (1953) 63-141; and 18 (1954), 27-81.

[26] A. Selberg, Note on a paper of L. G. Sathe, J. Indian Math. Soc. 18 (1954) 83-87.

[27] G. Tenenbaum, Introduction to Analytic and Probabilistic Number Theory, Cambridge Stud. Adv. Math., vol. 46, Cambridge Univ. Press, 1995. Third Edition, in French, Échelles Belin, (2008).

[28] _ Sur la probabilité qu'un entier possède un diviseur dans un intervalle donné, Compositio Math. 51 (1984), 243-263.

\section{R. C. Vaughan}

\section{The Hardy-Littlewood-Ramanujan Method}

In modern analytic number theory there are three very powerful standard techniques that are central to many investigations. One is the use of the theory of Dirichlet series, including the Riemann zeta function and Dirichlet $L$-functions; a second is the application of sieve theory in all its manifestations; and the third is the subject of this survey. It has its genesis in two papers of Ramanujan. The most famous of these is the celebrated paper [Hardy, G. H. \& Ramanujan, S. 1918] (there are also announcements in [Hardy, G. H. \& Ramanujan, S. 1917a] and [Hardy, G. H. \& Ramanujan, S. 1917b]), which is concerned mostly with the partition function, but the paper [Ramanujan, S. 1918] also describes the same fundamental idea. This is that interesting number theoretic functions have representations, or close approximations, as infinite series indexed by the positive rational numbers-that is, the $a / q$ with $1 \leq a \leq q$ and $(a, q)=1$-and moreover that such expressions can be obtained by consideration of generating functions that have the unit circle as a natural boundary and the terms in the aforementioned series arise from the neighborhood of singularities

R. C. Vaughan is professor of mathematics at Pennsylvania State University. His email address is rvaughan@math. psu.edu.

Research supported by NSA grant number H98230-12-10276 . 
at $e(a / q)$. A very simple example of this is the interesting formula

$$
\sigma(n)=\frac{\pi^{2}}{6} n \sum_{q=1}^{\infty} q^{-2} c_{q}(n),
$$

which first appears in the second of these papers. Here $c_{q}(n)$ is the eponymous sum

$$
c_{q}(n)=\sum_{\substack{a=1 \\(a, q)=1}}^{q} e(a n / q) .
$$

In addition to the partition function, Hardy and Ramanujan also observe that the same ideas can be applied to the number of representations of a number as a sum of a fixed number of squares.

One might speculate how this could have developed if Ramanujan had not been struck down by illness. Fortunately, Hardy and Littlewood realized the very great importance and generality of the underlying conceptions and were able to develop them so as to apply to the Goldbach and Waring, and cognate, problems. There was also the realization of the predictive possibilities of the method in those cases where the arguments could not be otherwise pursued to a satisfactory conclusion. Later this led, through further important ideas of I. M. Vinogradov and Davenport, in the 1930s to the essential resolution of the ternary Goldbach problem (namely, that every large odd integer is the sum of three primes) and substantial progress in the known upper bounds for $G(k)$ (the smallest $s$ such that every large natural number is the sum of at most $s k$ th powers) in Waring's problem (see [Vaughan, R. C. 1997]). Davenport, along with Birch and Lewis, embarked on the question of applying the method to nondiagonal questions, for example, the nontrivial representation of zero by cubic forms (see [Davenport, H. 1963] and [Davenport, H. 1962, 2005]). [Davenport, H. \& Lewis, D. J. 1963] had also established the Artin conjecture concerning the local-to-global principle for diagonal forms for all but a finite set of degrees. When I was a postgraduate student in the late 1960s, I became interested in the method. However, I was warned by a number of distinguished mathematicians that the method was already played out and it would be better to work on other things! Nevertheless, in [Vaughan, R. C. 1977] I did manage to use the method to deal with the cases not dealt with by Davenport and Lewis, and in a different direction [Montgomery, H. L. \& Vaughan, R. C. 1975] we were able to establish that there is a positive number $\delta$ such that the number $E(x)$ of even numbers not exceeding $x$ which are not the sum of two primes satisfies

$$
E(x) \ll x^{1-\delta} .
$$

In the last twenty-five years there has been a huge explosion of activity. This note is too short to do more than mention a few highlights, with the hope that the reader may be pointed in an interesting direction. On Waring's problem the development of efficient differencing, building on the earlier work, especially that of Davenport, led to significant further progress on the upper bound for $G(k)$, and there is a survey of this and some other connected results in [Vaughan, R. C. \& Wooley, T. D. 2002]. Very recently, the sensational work of [Wooley, T. D. to appear] now means that the asymptotic formula for the number of representations of a natural number as the sum of $s k$ th powers is known to hold when $s \geq 2 k^{2}-2 k-8$. On questions involving forms there has been impressive progress on applications of the method on a number of fronts, especially by [Schmidt, W. M. 1979] and [Wooley, T. D. 1997] on small zeros; [Heath-Brown, D. R. 1983], [Hooley, C., 1988, 1991, 1994], and [Hooley, C., 1988, 1991, 1994] on cubic forms; [Heath-Brown, D. R. \& Browning, T. D. 2009] on cubic polynomials; and [Brüdern, J \& Wooley, T. D. 1998] and [Brüdern, J \& Wooley, T. D. 2003] on a variety of topics.

In a different direction, [Heath-Brown, D. R. 1981] has ingeniously combined the circle method with sieve theory, and this was taken up by [Brüdern, J. 1988] in his thesis and has been exploited extensively by [Brüdern, J. 1995], [Brüdern, J. \& Kawada, K. 2002], [Kawada, K. 1997], and [Kawada, K. 2005]. This has spawned a whole new industry of ongoing work on sums of powers of primes and almost primes.

In the original series of papers "On some problems of partitio numerorum" by Hardy and Littlewood there was an unpublished manuscript on small differences between consecutive primes, and it was this manuscript which, when combined with developments in the large sieve, led to the work of [Bombieri, E. \& Davenport, H. 1966] and the later refinements of [Pil'tjaŭ, G. Z. 1972], [Huxley, M. N. 1977], and [Maier, H. 1985] on

$$
\delta=\liminf _{n \rightarrow \infty} \frac{p_{n+1}-p_{n}}{\log p_{n}} .
$$

The later proof by [Goldston, D. A., Pintz, J. \& Yildırım 2009] that $\delta=0$ rests mostly on sieve theory, yet the motivation for the shape of some of the main terms comes from heuristics arising from the Hardy-Littlewood method. In many ways the motivation for the large sieve also comes from the Hardy-Littlewood method, and perhaps this can be seen most clearly in [Gallagher, P. X. 1967]'s proof of the large sieve inequality.

An unexpected development was the adaptation of the method to deal with questions involving 
arithmetic progressions in general sets. This began with the seminal paper of [Roth, K. F. 1953] dealing with sets having no three elements in arithmetic progression and led to [Gowers, W. T. 1998]'s proof of Szemerédi's theorem and the theorem of [Green, B. J. \& Tao, T. 2008] that the primes contain arbitrarily long arithmetic progressions. This is another very active area; see, for example, [Green, B. J. 2007] or [Tao, T. \& Vu, Van, 2006]. A cognate area concerns the mean square distribution of sets of integers in arithmetic progressions. The prototype is the more precise version of the Barban-Davenport-Halberstam theorem concerning the set of primes due to [Montgomery, H. L. 1970] and refined by [Hooley, C. 1975]. Montgomery used a variant of the Hardy-Littlewood method, and more recently this was revived by [Goldston, D. A. \& Vaughan, R. C. 1996] for the set of primes and by [Vaughan, R. C. 1998] to treat general dense sets. This has been taken a step further by [Brüdern, J. 2009], which extends the ideas of the latter paper to treat general binary additive problems.

Another aspect of the method is that the idea of large peaks near "rational points" $e(a / q)$ with $q$ relatively small (the major arcs) and lesser peaks near $e(a / q)$ with $q$ relatively large (the minor arcs) has suggested divisions of arguments in, apparently, otherwise quite unrelated topics. An important example of this is the work of Bombieri, Iwaniec, Huxley, and Watt on exponential sums which led to the currently best-known bounds for the Riemann zeta function on the $\frac{1}{2}$-line, and the error terms in the Dirichlet divisor problem and Gauss's estimation of the number of lattice points in a large disc centered at the origin. For an exposition of this, see [Huxley, M. N. 1996].

For standard accounts of the Hardy-Littlewood method in its more classical forms, see [Davenport, H. 1962, 2005] and [Vaughan, R. C. 1997]. The foreword to the second edition of [Davenport, H. 1962, 2005] gives a good survey of a number of the applications as they stood in 2005.

\section{References}

[Bombieri, E. \& Davenport, H. 1966] Small difference between prime numbers, Proc. Royal Soc. London 293A (1966), 1-18.

[Brüdern, J. 1988] Iterationsmethoden in der additiven Zahlentheoerie, Thesis, Göttingen, 1988.

[Brüdern, J. 1995] A sieve approach to the WaringGoldbach problem. I: Sums of four cubes, Ann. Scient. École. Norm. Sup. (4) 28 (1995), 461-476.

[Brüdern, J. 2009] Binary additive problems and the circle method, multiplicative sequences and convergent sets, Analytic Number Theory, Essays in Honour of Klaus Roth, 2009, Cambridge University Press, pp. 91-132.
[Brüdern, J. \& Kawada, K. 2002] Ternary problems in additive prime number theory, Analytic Number Theory (Chaohua Jia and Kohji Matsumoto, eds.), Kluwer, 2002, pp. 39-91.

[Brüdern, J \& Wooley, T. D. 1998] The addition of binary cubic forms, Philos. Trans. Roy. Soc. London Ser. A 356 (1998), 701-737.

[Brüdern, J \& Wooley, T. D. 2003] The paucity problem for certain pairs of diagonal equations, Quart. J. Math. 54 (2003), 41-48.

[Davenport, H. 1962, 2005] Analytic Methods for Diophantine Equations and Diophantine Inequalities, First edition, Ann Arbor Publishers, 1962; Second edition, Cambridge University Press, 2005.

[Davenport, H. 1963] Cubic forms in sixteen variables, Proc. Royal Soc. London 272A (1963), 285-303.

[Davenport, H. \& Lewis, D. J. 1963] Homogeneous additive equations, Proc. Royal Soc. London 274A (1963), 443-460.

[Gallagher, P. X. 1967] The large sieve, Mathematika 14 (1967), 14-20.

[Goldston, D. A., Pintz, J. \& Ylldırım 2009] Primes in tuples. I, Annals of Mathematics 170 (2009), 819-862.

[Goldston, D. A. \& Vaughan, R. C. 1996] On the Montgomery-Hooley asymptotic formula, Sieve Methods, Exponential Sums, and Their Application in Number Theory, Greaves, Harman, Huxley (eds.), Cambridge University Press, 1996, pp. 117-142.

[Green, B. J. 2007] Generalizing the Hardy-Littlewood method for primes, Proc. ICM (Madrid, 2006), vol. 2, European Math. Soc., 2007, pp. 373-399.

[Green, B. J. \& Tao, T. 2008] The primes contain arbitrarily long arithmetic progressions, Ann. Math. 167 (2008), 481-547.

[Gowers, W. T. 1998] A new proof of Szemerédi's theorem for progressions, Geom. Funct. Anal. 8 (1998), 529551.

[Hardy, G. H. \& Ramanujan, S. 1917a] Une formule asymptotique pour le nombre des partitions de $n$, Comptes Rendus (January 2, 1917).

[Hardy, G. H. \& Ramanujan, S. 1917b] Asymptotic formulæ in combinatory analysis, Proc. London Math. Soc. 2 (1917), records for March 1, 1917.

[Hardy, G. H. \& Ramanujan, S. 1918] Asymptotic formulæ in combinatory analysis, Proc. London Math. Soc. 2 (1918), 75-115.

[Heath-Brown, D. R. 1981] Three primes and an almost prime in arithmetic progression, J. London Math. Soc. (2) 23 (1981), 396-414.

[Heath-Brown, D. R. 1983] Cubic forms in ten variables, Proc. London Math. Soc. (3) 47 (1983), 225-257.

[Heath-Brown, D. R. 2007] Cubic forms in 14 variables, Inventiones Mathematicae 170 (2007), 199-230.

[Heath-Brown, D. R. \& Browning, T. D. 2009] Integral points on cubic hypersurfaces, Analytic Number Theory, Essays in Honour of Klaus Roth, Cambridge University Press, 2009, 75-90.

[Hooley, C. 1975] On the Barban-Davenport-Halberstam theorem. I, J. Reine Angew. Math. 274/275 (1975), 206-223.

[Hooley, C., 1988, 1991, 1994] On nonary cubic forms. I, II, III, J. Reine Angew. Math. 386 (1988), 32-98; 415 (1991), 95-165; 456 (1994), 53-63.

[Huxley, M. N. 1977] Small differences between consecutive primes. II, Mathematika 24 (1977), 142-152. 
[Huxley, M. N. 1996] Area, Lattice Points, and Exponential Sums, London Math. Soc. Monographs, Oxford University Press, 1996.

[Kawada, K. 1997] Note on the sum of cubes of primes and an almost prime, Arch. Math. 69 (1997), 13-19.

[Kawada, K. 2005] On sums of seven cubes of almost primes, Acta Arith. 117 (2005), 213-245.

[Maier, H. 1985] Primes in short intervals, Michigan Math. J. 32 (1985), 221-225.

[Montgomery, H. L. 1970] Primes in arithmetic progressions, Michigan Math. J. 17 (1970), 33-39.

[Montgomery, H. L. \& Vaughan, R. C. 1975] The exceptional set in Goldbach's problem, Acta Arithmetica 27 (1975), 353-370.

[Pil'tjaĭ, G. Z. 1972] On the size of the difference between consecutive primes, Izdat. Saratov Univ., 1972, pp. 73-79.

[Ramanujan, S. 1918] On certain trigonometrical sums and their applications in the theory of numbers, Trans. Cambridge Phil. Soc. 13 (1918), 259-276.

[Roth, K. F. 1953] On certain sets of integers, J. London Math. Soc. 28 (1953), 245-252.

[Schmidt, W. M. 1979] Small zeros of additive forms in many variables. I, II, Trans. Amer. Math. Soc. 248 (1979), 121-133; Acta Math. 143 (1979), 219-232.

[Tao, T. \& Vu, Van, 2006] Additive Combinatorics, Cambridge University Press, 2006.

[Vaughan, R. C. 1977] Homogeneous additive equations and Waring's problem, Acta Arith. 33 (1977), 231-253.

[Vaughan, R. C. 1997] The Hardy-Littlewood Method, Cambridge Tracts in Mathematics, vol. 125, Cambridge University Press, 1997.

[Vaughan, R. C. 1998] On a variance associated with the distribution of general sequences in arithmetic progressions. I \& II, Philos. Trans. Royal Soc. Lond. Ser. A Math. Phys. Eng. Sci., 356 (1998), 781-791, 793-809.

[Vaughan, R. C. \& Wooley, T. D. 2002] Waring's Problem: A survey, Number Theory for the Millennium. III, A K Peters, 2002, pp. 301-340 (see also Surveys in Number Theory, A K Peters, 2003, pp. 285-324 (M. A. Bennett, B. C. Berndt, N. Boston, H. G. Diamond, A. J. Hildebrand, and W. Philipp, eds.).

[Wooley, T. D. 1997] Forms in many variables, Analytic Number Theory: Proceedings of the 39th Taniguchi International Symposium, Kyoto, May 1996 (Y. Motohashi, ed.), London Mathematical Society Lecture Notes, vol. 247, Cambridge University Press, Cambridge, 1997, pp. 361-376.

[Wooley, T. D.] Vinogradov's mean value theorem via efficient congruencing, II, Duke Math. J. (to appear).

\section{S. Ole Warnaar}

\section{Ramanujan's ${ }_{1} \psi_{1}$ Summation}

Notation. It is impossible to give an account of the ${ }_{1} \psi_{1}$ summation without introducing some $q$-series notation. To keep the presentation as simple as possible, we assume that $0<q<1$. Suppressing $q$-dependence, we define two $q$-shifted factorials: $(a)_{\infty}:=\prod_{k=0}^{\infty}\left(1-a q^{k}\right)$ and $(a)_{z}:=(a)_{\infty} /\left(a q^{Z}\right)_{\infty}$ for $z \in \mathbb{C}$. Note that $1 /(q)_{n}=0$ if $n$ is a negative integer. For $x \in \mathbb{C}-\{0,-1, \ldots\}$, the $q$-gamma function is defined as $\Gamma_{q}(x):=(q)_{x-1} /(1-q)^{x-1}$.

Ramanujan recorded his now famous ${ }_{1} \psi_{1}$ summation as item 17 of Chapter 16 in the second of his three notebooks [13, p. 32], [46]. It was brought to the attention of the wider mathematical community in 1940 by Hardy, who included it in his twelfth and final lecture on Ramanujan's work [31]. Hardy remarked that the result constituted "a remarkable formula with many parameters." Instead of presenting the ${ }_{1} \psi_{1}$ sum as given by Ramanujan and Hardy, we will state its modern form:

$$
\begin{aligned}
& \text { (1) } \sum_{n=-\infty}^{\infty} \frac{(a)_{n}}{(b)_{n}} z^{n}=\frac{(a z)_{\infty}(q / a z)_{\infty}(b / a)_{\infty}(q)_{\infty}}{(z)_{\infty}(b / a z)_{\infty}(q / a)_{\infty}(b)_{\infty}} \text {, } \\
& |b / a|<|z|<1 \text {, }
\end{aligned}
$$

where it is understood that $a, q / b \notin\left\{q, q^{2}, \ldots\right\}$. Characteristically, Ramanujan did not provide a proof of (1). Neither did Hardy, who, however, remarked that it could be "deduced from one which is familiar and probably goes back to Euler." The result to which Hardy was referring is another famous identity-known as the $q$ binomial theorem-corresponding to (1) with $b=$ $q: \sum_{n=0}^{\infty} z^{n}(a)_{n} /(q)_{n}=(a z)_{\infty} /(z)_{\infty}$ and valid for $|z|<1$. Although not actually due to Euler, the $q$-binomial theorem is certainly classic. It seems to have appeared first and without proof (for $a=q^{-N}$ ) in Rothe's 1811 book Systematisches Lehrbuch der Arithmetik, and in the 1840s many mathematicians of note, such as Cauchy (1843), Eisenstein (1846), Heine (1847), and Jacobi (1847), published proofs. The first proof of the $1 \psi_{1}$ sum is due to Hahn in 1949 [30] and, as hinted by Hardy, uses the $q$-binomial theorem. After Hahn, a large number of alternative proofs of (1) were found, including one probabilistic and three combinatorial proofs [2], [3], [5], [16], [17], [19],

S. Ole Warnaar is professor of mathematics at The University of Queensland. His email address is o.warnaar@maths.uq.edu.au.

The author thanks Dick Askey, Bruce Berndt, Susanna Fishel, Jeff Lagarias, and Michael Schlosser for their helpful correspondence.

This work was supported by the Australian Research Council. 
[20], [18], [23], [24], [32], [34], [35], [44], [48], [53], [50]. The proof from the book, which again relies on the $q$-binomial theorem, was discovered by Ismail [32] and is short enough to include here. Assuming $|z|<1$ and $|b|<\min \{1,|a z|\}$, both sides are analytic functions of $b$. Moreover, they coincide when $b=q^{k+1}$ with $k=0,1,2, \ldots$ by the $q$-binomial theorem with $a \mapsto a q^{-k}$. Since 0 is the accumulation point of this sequence of $b$ 's, the proof is complete.

Apart from the $q$-binomial theorem, the ${ }_{1} \psi_{1}$ sum generalizes another classic identity, known as the Jacobi triple-product identity: $\sum_{n=-\infty}^{\infty}(-1)^{n} z^{n} q^{\left(\begin{array}{c}n \\ 2\end{array}\right)}=(z)_{\infty}(q / z)_{\infty}(q)_{\infty}=: \theta(z)$. This result plays a central role in the theory of theta and elliptic functions.

\section{The ${ }_{1} \psi_{1}$ Sum as Discrete Beta Integral}

As pointed out by Askey [8], [9], the ${ }_{1} \psi_{1}$ summation may be viewed as a discrete analogue of Euler's beta integral. First, define the Jackson or $q$-integral $\int_{0}^{c \cdot \infty} f(t) \mathrm{d}_{q} t:=(1-q) \sum_{n=-\infty}^{\infty} f\left(c q^{n}\right) c q^{n}$. Replacing $(a, b, z) \mapsto\left(-c,-c q^{\alpha+\beta}, q^{\alpha}\right)$ in (1) then gives

(2) $\int_{0}^{c \cdot \infty} \frac{t^{\alpha-1}}{(-t)_{\alpha+\beta}} \mathrm{d}_{q} t=c^{\alpha} \frac{\theta\left(-c q^{\alpha}\right)}{\theta(-c)} \frac{\Gamma_{q}(\alpha) \Gamma_{q}(\beta)}{\Gamma_{q}(\alpha+\beta)}$,

where $\operatorname{Re}(\alpha), \operatorname{Re}(\beta)>0$. For real, positive $c$ the limit $q \rightarrow 1$ can be taken, resulting in the beta integral modulo the substitution $t \mapsto t /(1-t)$. Askey further noted in [8] that the specialization $(\alpha, \beta) \mapsto(x, 1-x)$ in (2) (so that $0<\operatorname{Re}(x)<1$ ) may be viewed as a $q$-analogue of Euler's reflection formula.

\section{Simple Applications of the ${ }_{1} \psi_{1}$ Sum}

There are numerous easy applications of the ${ }_{1} \psi_{1}$ sum. For example, Jacobi's well-known fourand six-square theorems as well as a number of similar results readily follow from (1); see e.g., [1], [14], [15], [21], [22], [25]. To give a flavor of how the ${ }_{1} \psi_{1}$ implies these types of results, we shall sketch a proof of the foursquare theorem. Let $r_{s}(n)$ be the number of representations of $n$ as the sum of $s$ squares. The generating function $R_{s}(q):=\sum_{n \geq 0} r_{s}(n)(-q)^{n}$ is given by $\left(\sum_{m=-\infty}^{\infty}(-1)^{m} q^{m^{2}}\right)^{s}$. By the tripleproduct identity this is also $\left((q)_{\infty} /(-q)_{\infty}\right)^{s}$. Any identity that allows the extraction of the coefficient of $(-q)^{n}$ results in an explicit formula for $r_{s}(n)$. Back to (1): replace $(b, z) \mapsto(a q, b)$ and multiply both sides by $(1-b) /(1-a b)$. By the geometric series this yields

$$
\text { (3) } \begin{aligned}
1+ & \frac{(1-a)(1-b)}{1-a b} \sum_{k, n=1}^{\infty} q^{k n}\left(a^{k} b^{n}-a^{-k} b^{-n}\right) \\
= & \frac{(a b q)_{\infty}(q / a b)_{\infty}(q)_{\infty}^{2}}{(a q)_{\infty}(q / a)_{\infty}(b q)_{\infty}(q / b)_{\infty}},
\end{aligned}
$$

which may also be found in Kronecker's 1881 paper "Zur Theorie der elliptischen Functionen". For $a, b \rightarrow-1$ the right side gives $R_{4}(q)$, whereas the left side becomes

$$
\begin{aligned}
1-8 & \sum_{m=1}^{\infty} q^{m} \sum_{\substack{n, k=1 \\
n k=m}}^{\infty} n(-1)^{n+k} \\
& =1+8 \sum_{m=1}^{\infty}(-q)^{m} \sum_{\substack{d \geq 1 \\
4 \nmid d \mid m}} d .
\end{aligned}
$$

Hence $r_{4}(n)=8 \sum_{d \geq 1 ; 4 \nmid d \mid n} d$. This result of Jacobi implies Lagrange's theorem that every positive integer is a sum of four squares. By taking $a, b^{2} \rightarrow-1$ in (3) the reader will have little trouble showing that $r_{2}(n)=4\left(d_{1}(n)-d_{3}(n)\right)$, with $d_{k}(n)$ the number of divisors of $n$ of the form $4 m+k$. This is a result of Gauss and Lagrange which implies Fermat's two-square theorem.

Other simple but important applications of the ${ }_{1} \psi_{1}$ sum concern orthogonal polynomials. In [11] it was employed by Askey and Wilson to compute a special case-corresponding to the continuous $q$ Jacobi polynomials-of the Askey-Wilson integral, and in [10] Askey gave an elementary proof of the full Askey-Wilson integral using the ${ }_{1} \psi_{1}$ sum. The sum also implies the norm evaluation of the weight functions of the $q$-Laguerre polynomials [45]. These form a family of orthogonal polynomials with discrete measure $\mu$ on $[0, c \cdot \infty)$ given by $\mathrm{d}_{q} \mu(t)=t^{\alpha} /(-t)_{\infty} \mathrm{d}_{q} t$. The normalization $\int \mathrm{d}_{q} \mu(t)$ thus follows from the $q$-beta integral (2) in the limit of large $\beta$.

\section{Generalizations in One Dimension}

There exist several generalizations of Ramanujan's sum containing one additional parameter. In his work on partial theta functions, Andrews [4] obtained a generalization in which each product of four infinite products on the right-hand side is replaced by six such products. Another example is the curious identity of Guo and Schlosser, which is no longer hypergeometric in nature [27]:

$$
\begin{aligned}
& \sum_{k=-\infty}^{\infty} \frac{(a)_{k}\left(1-a c_{k} q^{k}\right)\left(c_{k} q\right)_{\infty}\left(b / a c_{k}\right)_{\infty}}{(b)_{k}\left(1-a z q^{k}\right)\left(a c_{k}\right)_{\infty}\left(q / a c_{k}\right)_{\infty}} c_{k}^{k} \\
& =\frac{1}{(1-z)} \frac{(q)_{\infty}(b / a)_{\infty}}{(q / a)_{\infty}(b)_{\infty}},
\end{aligned}
$$

where $c_{k}:=z\left(1-a c z q^{k}\right) /\left(1-a z q^{k}\right)$ and $|b / a c|<$ $|z|<1$. For $c=1$ this is (1).

As discovered by Schlosser [49], a quite different extension of the ${ }_{1} \psi_{1}$ sum arises by considering noncommutative variables. Let $R$ be a unital Banach algebra with identity 1 , central elements $b$ and $q$, and norm $\|\cdot\|$. Write $a^{-1}$ for the inverse of an invertible element $a \in R$. Let $\prod_{i=m}^{n} a_{i}$ stand for 1 
if $n=m-1, a_{m} \cdots a_{n}$ if $n \geq m$ and $a_{m-1}^{-1} \cdots a_{n+1}^{-1}$ if $n<m-1$, and define

$$
\begin{aligned}
& \left(\begin{array}{l}
a_{1}, \ldots, a_{r} \\
b_{1}, \ldots, b_{r}
\end{array} z\right)_{k}^{ \pm} \\
& \quad:=\prod_{i}\left[z \prod_{s=1}^{r}\left(1-a_{s} q^{i-1}\right)\left(1-b_{s} q^{i-1}\right)^{-1}\right],
\end{aligned}
$$

where $k \in \mathbb{Z} \cup\{\infty\}, a_{1}, \ldots, a_{r}, b_{1}, \ldots, b_{r} \in R, \prod_{i}=$ $\prod_{i=1}^{k}$ in the + case and $\prod_{i}=\prod_{i=k}^{1}$ in the case. Subject to $\max \left\{\|q\|,\|z\|,\left\|b a^{-1} z^{-1}\right\|\right\}<1$, the following noncommutative ${ }_{1} \psi_{1}$ sum holds:

$$
\begin{aligned}
& \sum_{k=-\infty}^{\infty}\left(\begin{array}{l}
a \\
b
\end{array} ; z\right)_{k}^{+} \\
& =\left(\begin{array}{c}
z a \\
z
\end{array} ; 1\right)_{\infty}^{-}\left(\begin{array}{c}
q a^{-1} z^{-1} \\
q z a^{-1} z^{-1}
\end{array} ; 1\right)_{\infty}^{+}\left(\begin{array}{c}
b z a^{-1} z^{-1}, q \\
b a^{-1} z^{-1}, b
\end{array} ; 1\right)_{\infty}^{-} .
\end{aligned}
$$

\section{Higher-Dimensional Generalizations}

Various authors have generalized (1) to multiple ${ }_{1} \psi_{1}$ sums. Below we state a generalization due to Gustafson and Milne [28], [41] which is labelled by the A-type root system. Similar such ${ }_{1} \psi_{1}$ sums are given in [6], [7], [29], [43], [47]. More involved multiple ${ }_{1} \psi_{1}$ sums with a Schur or Macdonald polynomial argument can be found in [12], [36], [42], [52]. For $r=\left(r_{1}, \ldots, r_{n}\right) \in \mathbb{Z}^{n}$ denote $|r|:=r_{1}+\cdots+r_{n}$. Then

$$
\begin{gathered}
\sum_{r \in \mathbb{Z}^{n}} z^{|r|} \prod_{1 \leq i<j \leq n} \frac{x_{i} q^{r_{i}}-x_{j} q^{r_{j}}}{x_{i}-x_{j}} \prod_{i, j=1}^{n} \frac{\left(a_{j} x_{i j}\right)_{r_{i}}}{\left(b_{j} x_{i j}\right)_{r_{i}}} \\
=\frac{(a z)_{\infty}(q / a z)_{\infty}}{(z)_{\infty}(b / a z)_{\infty}} \prod_{i, j=1}^{n} \frac{\left(b_{j} x_{i j} / a_{i}\right)_{\infty}\left(q x_{i j}\right)_{\infty}}{\left(q x_{i j} / a_{i}\right)_{\infty}\left(b_{j} x_{i j}\right)_{\infty}},
\end{gathered}
$$

where $a:=a_{1} \cdots a_{n}, b:=q^{1-n} b_{1} \cdots b_{n}, x_{i j}:=$ $x_{i} / x_{j}$ and $|b / a|<|z|<1$. Milne first proved this for $b_{1}=\cdots=b_{n}$ [41], and shortly thereafter Gustafson established the full result [28]. We have already seen that the ${ }_{1} \psi_{1}$ sum implies the Jacobi triple-product identity. The latter is the $\mathrm{A}_{1}^{(1)}$ case of Macdonald's generalized Weyl denominator identities for affine root systems [38]. To obtain further Macdonald identities from the GustafsonMilne sum, one replaces $z \rightarrow z / a$ before letting $a_{1}, \ldots, a_{n} \rightarrow \infty$ and $b_{1}, \ldots, b_{n} \rightarrow 0$. Extracting the coefficient of $z^{0}$ (on the right this requires the triple-product identity) results in the Macdonald identity for $\mathrm{A}_{n-1}^{(1)}$.

Higher-dimensional generalizations of a special case of the ${ }_{1} \psi_{1}$ sum can be given for all affine root systems. A full description is beyond our scope here, so we will sketch only the simplest case. The reader is referred to [26], [38], [39], [40] for the full details. In [39] Macdonald gave the following multivariable extension of the product formula for the Poincaré polynomial of a Coxeter group:

(4)

$W(\boldsymbol{t}):=\sum_{w \in W} \prod_{\alpha \in R^{+}} \frac{1-t_{\alpha} \mathrm{e}^{w(\alpha)}}{1-\mathrm{e}^{w(\alpha)}}=\prod_{\alpha \in R^{+}} \frac{1-t_{\alpha} \boldsymbol{t}^{\mathrm{ht}(\alpha)}}{1-\boldsymbol{t}^{\mathrm{ht}(\alpha)}}$.

Here $R$ is a reduced, irreducible finite root system in a Euclidean space $V, R^{+}$the set of positive roots, $W$ the Weyl group, and $t_{\alpha}$ for $\alpha \in R^{+}$a set of formal variables constant along Weyl orbits. The symbol $\boldsymbol{t}^{\text {ht }(\alpha)}$ stands for $\prod_{\beta \in R^{+}} t_{\beta}^{(\beta, \alpha) /\|\alpha\|^{2}}$ with $(\cdot, \cdot)$ the $W$-invariant positive definite bilinear form on $V$. If all $t_{\alpha}$ are set to $t$, then $t^{\mathrm{ht}(\alpha)}=t^{\mathrm{ht}(\alpha)}$ with $\mathrm{ht}(\alpha)$ the usual height function on $R$, in which case $W(\boldsymbol{t})$ reduces to the classical Poincaré polynomial $W(t)$. Now let $S$ be a reduced, irreducible affine root system of type $S=S(R)$ [38]. In analogy with the finite case, assume that $t_{a}$ for $a \in S$ is constant along orbits of the affine Weyl group $W$ of $S$. Then Macdonald generalized (4) to [40]

$$
\text { (5) } \begin{aligned}
\sum_{w \in W} & \prod_{a \in S^{+}} \frac{1-t_{a} \mathrm{e}^{w(a)}}{1-\mathrm{e}^{w(a)}} \\
= & \prod_{\alpha \in R^{+}} \frac{\left(t_{\alpha} \boldsymbol{t}^{\mathrm{ht}(\alpha)}\right)_{\infty}\left(\boldsymbol{t}^{\mathrm{ht}(\alpha)} q^{\chi(\alpha \in B)} / t_{\alpha}\right)_{\infty}}{\left(\boldsymbol{t}^{\mathrm{ht}(\alpha)}\right)_{\infty}^{2}},
\end{aligned}
$$

where $B$ is a base for $R$. The parameter $q$ on the right is fixed by $q=\prod_{a \in B(S)} \exp \left(n_{a} a\right)$, where $B(S)$ is a basis for $S$ and the $n_{a}$ are the labels of the extended Dynkin diagrams given in [38]. If $R$ is simply-laced, then $t_{a}=t$. In the case of $S(R)=\mathrm{A}_{1}^{(1)}, q=\exp \left(a_{0}+a_{1}\right)$ so that after replacing $\exp \left(a_{1}\right)$ by $x$ we obtain the ${ }_{1} \psi_{1}$ sum (1) with $(a, b, z) \rightarrow(x / t, t x, t)$. This is not the end of the story concerning root systems and the ${ }_{1} \psi_{1}$ sum. Identity (5) can be rewritten as [40]

(6)

$$
\begin{aligned}
\sum_{\gamma \in Q^{\vee}} & \prod_{\alpha \in R} \frac{\left(q \mathrm{e}^{\alpha}\right)_{(\alpha, \gamma)}}{\left(t_{\alpha} q \mathrm{e}^{\alpha}\right)_{(\alpha, \gamma)}} \\
= & \prod_{\alpha \in R^{+}} \frac{\left(t_{\alpha} \boldsymbol{t}^{\mathrm{ht}(\alpha)} q\right)_{\infty}\left(\boldsymbol{t}^{\mathrm{ht}(\alpha)} q^{\alpha(\alpha \in B)} / t_{\alpha}\right)_{\infty}}{\left(\boldsymbol{t}^{\mathrm{ht}(\alpha)} q\right)_{\infty}\left(\boldsymbol{t}^{\mathrm{ht}(\alpha)}\right)_{\infty}} \\
& \times \frac{\left(q \mathrm{e}^{\alpha}\right)_{\infty}\left(q \mathrm{e}^{-\alpha}\right)_{\infty}}{\left(t_{\alpha} q \mathrm{e}^{\alpha}\right)_{\infty}\left(t_{\alpha} q \mathrm{e}^{-\alpha}\right)_{\infty}},
\end{aligned}
$$

where $Q^{\vee}$ is the coroot lattice. Interestingly, for $t_{\alpha}=t$ this was also found by Fishel, Grojnowski and Teleman [26] by computing the generating function of the $q$-weighted Euler characteristics of certain Dolbeault cohomologies. For $R=\mathrm{A}_{n-1}, Q^{\vee}=Q=\sum_{i=1}^{n} r_{i} \epsilon_{i}$ with $|r|=0$, $R=\left\{\epsilon_{i}-\epsilon_{j}: 1 \leq i \neq j \leq n\right\}$, and $t^{\text {ht }\left(\epsilon_{i}-\epsilon_{j}\right)}=t^{j-i}$. By fairly elementary manipulations, the identity (6) may then be transformed into the multiple ${ }_{1} \psi_{1}$ sum 


$$
\begin{aligned}
& \sum_{r \in \mathbb{Z}^{n}} z^{|r|} \frac{(a)_{|r|}}{(b)_{|r|}} \prod_{1 \leq i<j \leq n} \frac{x_{i} q^{r_{i}}-x_{j} q^{r_{j}}}{x_{i}-x_{j}} \frac{\left(t^{-1} x_{i j}\right)_{r_{i}-r_{j}}}{\left(t q x_{i j}\right)_{r_{i}-r_{j}}} t^{r_{i}-r_{j}} q^{-r_{j}} \\
& =\frac{(a z)_{\infty}(q / a z)_{\infty}(b / a)_{\infty}(t q)_{\infty}}{(z)_{\infty}(b / a z)_{\infty}(q / a)_{\infty}(b)_{\infty}} \prod_{i=1}^{n-1} \frac{\left(t^{i+1} q\right)_{\infty}}{\left(t^{i}\right)_{\infty}} \prod_{i, j=1}^{n} \frac{\left(q x_{i j}\right)_{\infty}}{\left(t q x_{i j}\right)_{\infty}}
\end{aligned}
$$

for $|b / a|<|z|<1$ and $|t|<1$. This is the only result in this survey that is new.

We finally remark that all higher-dimensional ${ }_{1} \psi_{1}$ sums admit representations as discrete Selbergtype integrals. The most important such integrals are due to Aomoto [6], [7] and Ito [33], and are closely related to (5). Further examples may be found in [37], [51].

\section{References}

[1] C. ADIGA, On the representations of an integer as a sum of two or four triangular numbers, Nihonkai Math. J. 3 (1992), 125-131.

[2] C. Adiga, B. C. Berndt, S. Bhargava, and G. N. WATSON, Chapter 16 of Ramanujan's second notebook: theta-functions and $q$-series, Mem. Amer. Math. Soc. 53 (1985), no. 315.

[3] G. E. Andrews, On Ramanujan's ${ }_{1} \psi_{1}(a ; b ; z)$, Proc. Amer. Math. Soc. 22 (1969), 552-553.

[4] _ , Ramanujan's “lost” notebook. I. Partial $\theta$-functions, Adv. in Math. 41 (1981), 137-172.

[5] G. E. ANDrEws and R. AsKeY, A simple proof of Ramanujan's $1 \psi_{1}$, Aequationes Math. 18 (1978), 333-337.

[6] K. Аомото, On a theta product formula for the symmetric A-type connection function, Osaka J. Math. 32 (1995), 35-39.

[7] _ , On elliptic product formulas for Jackson integrals associated with reduced root systems, $J$. Algebraic Combin. 8 (1998), 115-126.

[8] R. ASKeY, The $q$-gamma and $q$-beta functions, Applicable Anal. 8 (1978/79), 125-141.

[9] __ Ramanujan's extensions of the gamma and beta functions, Amer. Math. Monthly 87 (1980), 346359.

[10] _ An elementary evaluation of a beta type integral, Indian J. Pure Appl. Math. 14 (1983), 892-895.

[11] R. ASKEY and J. WILSON, Some basic hypergeometric orthogonal polynomials that generalize Jacobi polynomials, Mem. Amer. Math. Soc. 54 (1985), no. 319.

[12] T. H. BAKER and P. J. FORRESTER, Transformation formulas for multivariable basic hypergeometric series, Methods Appl. Anal. 6 (1999), 147-164.

[13] B. C. BERndT, Ramanujan's Notebooks. Part III, Springer-Verlag, New York, 1991.

[14] S. BHARGAVA and C. ADIGA, Simple proofs of Jacobi's two and four square theorems, Int. J. Math. Ed. Sci. Tech. 19 (1988), 779-782.

[15] S. H. CHAN, An elementary proof of Jacobi's six squares theorem, Amer. Math. Monthly 111 (2004), 806-811.

[16] _ A short proof of Ramanujan's famous ${ }_{1} \psi_{1}$ summation formula, J. Approx. Theory 132 (2005), $149-153$.

[17] S. H. L. Chen, W. Y. C. Chen, A. M. Fu, and W. J. T. ZAnG, The Algorithm Z and Ramanujan's ${ }_{1} \psi_{1}$ summation, Ramanujan J. 25 (2011), 37-47.

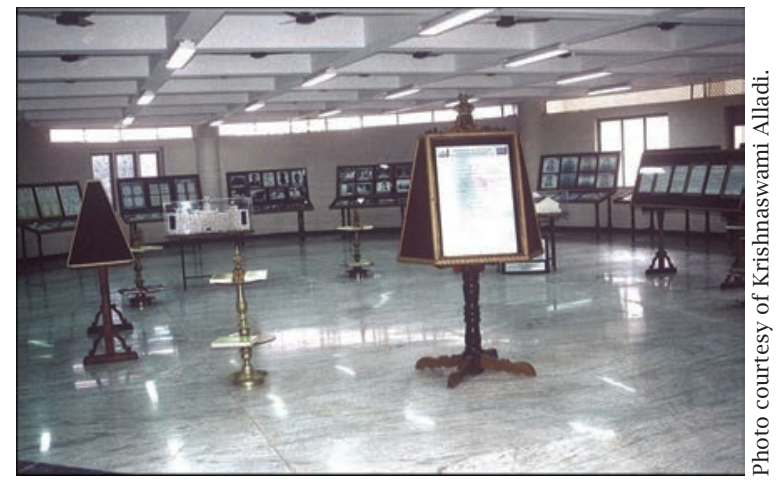

The museum at SASTRA University in Kumbakonam, where several manuscripts, letters, papers, documents, photographs, etc., relating to Ramanujan are displayed.

[18] W. Y. C. CHEN, and E. X. W. XIA, The $q$-WZ method for infinite series, J. Symbolic Comput. 44 (2009), 960-971.

[19] X. CHEN, Q. LIU and X. MA, A short proof of Ramanujan's ${ }_{1} \psi_{1}$ formula, J. Math. Res. Exposition 28 (2008), 46-48.

[20] W. CHU, Abel's lemma on summation by parts and Ramanujan's $1 \psi_{1}$-series identity, Aequationes Math. 72 (2006), 172-176.

[21] S. COOPER, On sums of an even number of squares, and an even number of triangular numbers: an elementary approach based on Ramanujan's ${ }_{1} \psi_{1}$ summation formula, Contemp. Math., vol. 291, Amer. Math. Soc., Providence, RI, 2001, pp. 115-137.

[22] S. COOPER and H. Y. LAM, Sums of two, four, six and eight squares and triangular numbers: an elementary approach, Indian J. Math. 44 (2002), 21-40.

[23] S. CoRTeEL, Particle seas and basic hypergeometric series, Adv. Appl. Math. 31 (2003), 199-214.

[24] S. CorTEel and J. LOVEJOY, Frobenius partitions and the combinatorics of Ramanujan's ${ }_{1} \psi_{1}$ summation, J. Combin. Theory Ser. A 97 (2002), 177-183.

[25] N. J. FInE, Basic Hypergeometric Series and Applications, Mathematical Surveys and Monographs, vol. 27, Amer. Math. Soc., Providence, RI, 1988.

[26] S. Fishel, I. GRojNowski, and C. TELEMAN, The strong Macdonald conjecture and Hodge theory on the loop Grassmannian, Ann. of Math. (2) 168 (2008), 175-220.

[27] V. J. Guo and M. J. Schlosser, Curious extensions of Ramanujan's ${ }_{1} \psi_{1}$ summation formula, J. Math. Anal. Appl. 334 (2007), 393-403.

[28] R. A. GuSTAFson, Multilateral summation theorems for ordinary and basic hypergeometric series in $U(n)$, SIAM J. Math. Anal. 18 (1987), 1576-1596.

[29] R. A. Gustafson and C. KRATtenthaler, Heine transformations for a new kind of basic hypergeometric series in $U(n)$, J. Comput. Appl. Math. 68 (1996), 151-158.

[30] W. HAHN, Beiträge zur Theorie der Heineschen Reihen, Math. Nachr. 2 (1949), 340-379.

[31] G. H. HARDY, Ramanujan. Twelve Lectures on Subjects Suggested by His Life and Work, Cambridge University Press, Cambridge, UK; Macmillan Company, New York, 1940.

[32] M. E. H. IsmaIL, A simple proof of Ramanujan's ${ }_{1} \psi_{1}$ sum, Proc. Amer. Math. Soc. 63 (1977), 185-186. 


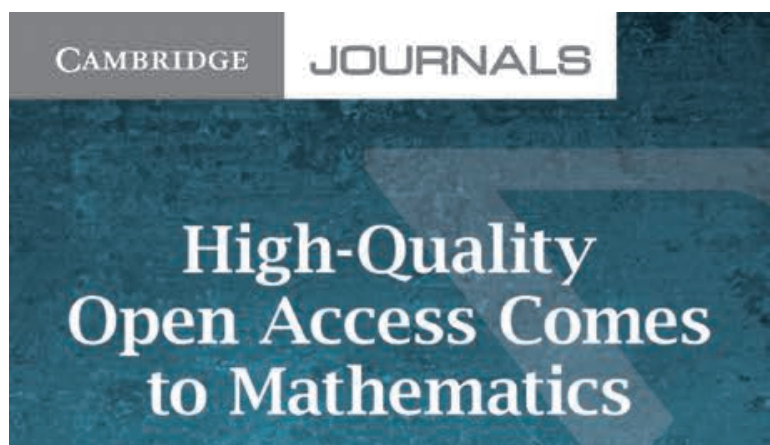

journals.cambridge.org/forumofmathematics

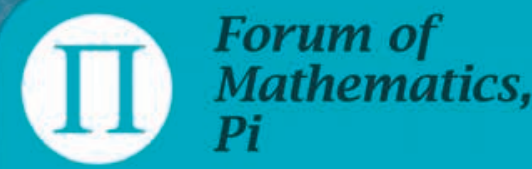

$P_{i}$ is the open access alternative to the leading generalist mathematics journals. Papers published will be of a high quality and of real interest to a broad cross-section of all mathematicians.

Submit your paper

journals.cambridge.org/pi/submit

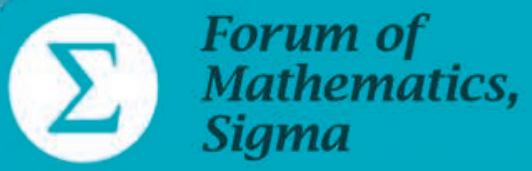

Sigma is an open access journal that will publish the best specialist research in your field. Editorial decisions are made by dedicated clusters of editors concentrated in the following areas:

foundations of mathematics $\bullet$ discrete mathematics $\bullet$ algebra $\bullet$ number theory - algebraic and complex geometry $\bullet$ differential geometry and geometric analysis $\bullet$ topology $\bullet$ analysis $\bullet$ probability and stochastics $\bullet$ differential equations - computational mathematics $\bullet$ applied analysis $\bullet$ mathematical physics $\bullet$ theoretical computer science Submit your paper journals.cambridge.org/sigma/submit
[33] M. ITO, On a theta product formula for Jackson integrals associated with root systems of rank two, $J$. Math. Anal. Appl. 216 (1997), 122-163.

[34] M. JACKSON, On Lerch's transcendent and the basic bilateral hypergeometric series $2 \psi_{2}, J$. London Math Soc. 25 (1950), 189-196.

[35] K. W. J. KADELL, A probabilistic proof of Ramanujan's $1 \psi_{1}$ sum, Siam. J. Math. Anal. 18 (1987), 1539-1548.

[36] J. KANEKO, A $1 \psi_{1}$ summation theorem for Macdonald polynomials, Ramanujan J. 2 (1996), 379-386.

[37] J. KANEKO, $q$-Selberg integrals and Macdonald polynomials, Ann. Sci. École Norm. Sup. (4) 29 (1996), 583-637.

[38] I. G. MACDONALD, Affine root systems and Dedekind's $\eta$-function, Invent. Math. 15 (1972), 91-143.

[39] _ The Poincaré series of a Coxeter group, Math. Ann. 199 (1972), 161-174.

[40] _ A formal identity for affine root systems, in Lie Groups and Symmetric Spaces, Amer. Math. Soc. Transl. Ser. 2, vol. 210, Amer. Math. Soc., Providence, RI, pp. 195-211, 2003.

[41] S. C. MiLne, A $U(n)$ generalization of Ramanujan's ${ }_{1} \psi_{1}$ summation, J. Math. Anal. Appl. 118 (1986), 263-277.

[42] _ Summation theorems for basic hypergeometric series of Schur function argument, in Progress in approximation theory, Springer Ser. Comput. Math., vol. 19, Springer, New York, 1992, pp. 51-77.

[43] S. C. Milne and M. SChlosser, A new $A_{n}$ extension of Ramanujan's ${ }_{1} \psi_{1}$ summation with applications to multilateral $\mathrm{A}_{n}$ series, Rocky Mountain J. Math. 32 (2002), 759-792.

[44] K. MimaCHI, A proof of Ramanujan's identity by use of loop integrals, SIAM J. Math. Anal. 19 (1988), 14901493.

[45] D. S. MoAK, The $q$-analogue of the Laguerre polynomials, J. Math. Anal. Appl. 81 (1981), 20-47.

[46] S. Ramanujan, Notebooks, Tata Institute of Fundamental Research, Bombay, 1957.

[47] M. SCHLOSSER, Summation theorems for multidimensional basic hypergeometric series by determinant evaluations, Discrete Math. 210 (2000), 151-169.

[48] _ Abel-Rothe type generalizations of Jacobi's triple product identity, in Theory and Applications of Special Functions, Dev. Math., vol. 13, Springer, New York, 2005, pp. 383-400.

[49] _ Noncommutative extensions of Ramanujan's ${ }_{1} \psi_{1}$ summation, Electron. Trans. Numer. Anal. 24 (2006), 94-102.

[50] K. Venkatachaliengar and S. CoOper, Development of Elliptic Functions according to Ramanujan, Monographs in Number Theory, vol. 6, World Scientific, Singapore, 2012.

[51] S. O. WARNAAR, $q$-Selberg integrals and Macdonald polynomials, Ramanujan J. 10 (2005), 237-268.

[52] _ The $\mathfrak{s}_{3}$ Selberg integral, Adv. Math. 224 (2010), 499-524.

[53] A. J. YEE, Combinatorial proofs of Ramanujan's ${ }_{1} \psi_{1}$ summation and the $q$-Gauss summation, J. Combin. Theory Ser. A 105 (2004), 63-77. 